\title{
Preoperative 3-dimensional computed tomography lung simulation before video-assisted thoracoscopic anatomic segmentectomy for ground glass opacity in lung
}

\author{
Liang Xue ${ }^{1}$, Hong Fan ${ }^{1}$, Woda Shi ${ }^{2}$, Di Ge ${ }^{1}$, Yi Zhang ${ }^{1}$, Qun Wang ${ }^{1}$, Yunfeng Yuan ${ }^{1}$ \\ ${ }^{1}$ Department of Thoracic Surgery, Zhongshan Hospital, Fudan University, Shanghai 200032, China; ${ }^{2}$ Department of Cardio-Thoracic Surgery, \\ Yancheng Third People's Hospital, Yancheng 224001, China \\ Contributions: (I) Conception and design: Y Yuan; (II) Administrative support: Q Wang; (III) Provision of study materials or patients: Q Wang, D \\ Ge, H Fan; (IV) Collection and assembly of data: L Xue, W Shi, Y Zhang; (V) Data analysis and interpretation: L Xue; (VI) Manuscript writing: All \\ authors; (VII) Final approval of manuscript: All authors. \\ Correspondence to: Yunfeng Yuan. Department of Thoracic Surgery, Zhongshan Hospital, Fudan University, 180 Fenglin Road, Xuhui District, \\ Shanghai 200032, China. Email: yuan.yunfeng@zs-hospital.sh.cn.
}

Background: Three-dimensional (3D) simulation of pulmonary vessels and the space between the lesion and adjacent tissues may improve the safety and accuracy of video-assisted thoracoscopic surgery (VATS) for lung. The aim of this study was to evaluate the effect of $3 \mathrm{D}$ simulation on the outcome of VATS segmentectomy for ground glass opacity (GGO) in lung.

Methods: We retrospectively analyzed 68 cases of small $(\leq 2 \mathrm{~cm})$ GGO, which were diagnosed as cT1aN0M0 lung cancer, from May 1, 2016 to February 28, 2017 in our institute. All the patients underwent VATS segmentectomy. The patients were divided into “3D" group, 3D preoperative reconstruction simulation in 36 patients and "non-3D" group, 32 patients with only computed tomography (CT). Operation plans were firstly made by CT in all patients, then by $3 \mathrm{D}$ simulation only in $3 \mathrm{D}$ group. The clinical outcomes, including operation time, blood loss, resection margin distance, length of postoperative stay and postoperative complications were compared between the two groups.

Results: There were 21 male and 47 female analyzed, aging from 34 to 72 years (median 57). In 3D group, pathological result showed 8 cases of adenocarcinoma, 23 cases of microinvasive adenocarcinoma (MIA), 5 cases of adenocarcinoma in situ (AIS). In non-3D group, 18 cases of MIA, 9 cases of adenocarcinoma and 5 cases of AIS were diagnosed pathologically. The blood loss, postoperative hospital stay and the incidence of the postoperative complications were similar in both of the groups. There was no 30-day postoperative mortality in either group. The median operation time for the $3 \mathrm{D}$ group (111 minute) was shorter than non-3D group (139 minute) ( $\mathrm{P}=0.03)$. Seven cases $(19 \%)$ in $3 \mathrm{D}$ group changed the original operation plan according to the simulation result with the consideration of adequate resection margin distance. All cases in $3 \mathrm{D}$ group had adequate resection margin distance. Four cases $(13 \%)$ in non-3D group got inadequate resection margin distance, and more lung tissues than the original plan were then resected in these patients $(\mathrm{P}=0.04)$.

Conclusions: 3D preoperative simulation may be more precise in operation plan than CT scan and can significantly shorten the operation time in VATS segmentectomy for GGO in lung.

Keywords: Video-assisted thoracoscopic surgery (VATS); segmentectomy; lung cancer; three-dimensional simulation (3D simulation)

Submitted May 27, 2018. Accepted for publication Sep 26, 2018.

doi: $10.21037 /$ jtd.2018.10.126

View this article at: http://dx.doi.org/10.21037/jtd.2018.10.126 


\section{Introduction}

Lobectomy is still regarded as the first choice for stage I nonsmall cell lung cancer (NSCLC). A meta-analysis showed that survival for stage I NSCLC was significantly better after lobectomy than after segmentectomy (1). Due to widespread practice of low-dose helical computed tomography (CT) for screening in recent years, detection of very small NSCLC lesions containing ground glass opacity (GGO) component is more and more common. Interest is raised in defining the role of sub-lobar resection such as segmentectomy in the treatment of these small GGO lesions. Till now, according to the National Comprehensive Cancer Network (NCCN) guidelines, the indication for sublobar resection is a peripheral nodule $<2 \mathrm{~cm}$ in size with at least one of the following: (I) pure bronchioalveolar carcinoma histology; (II) nodule with $50 \%$ ground glass appearance on CT imaging; and (III) radiologic surveillance confirming a long doubling time (>400 days) (2). In a recent prospective follow up study, limited resection for selected small-sized lung cancer with GGOs was performed safely without any recurrence (3). Results of two meta-analyses $(4,5)$ suggested that anatomic segmentectomy with systematic hilar and mediastinal node dissection may be particularly effective for small peripheral tumors smaller than $2 \mathrm{~cm}$.

Meanwhile, video-assisted thoracoscopic (VATS) segmentectomy is challenging in patients with GGO lesions. In addition to the thorough understanding of the complicated anatomical variations of segmental bronchi and vessels, the confirmation of the unpalpable GGO locations and accurate surgical plan for safe resection margins are also important for the ideal oncological treatment results.

Multi-detector CT can provide sources for the construction of three-dimensional (3D) images. 3D simulation technology was reported to be effectively applied to lobectomy and segmentectomy $(6,7)$. We supposed that 3D displays of GGO lesions and anatomic structures of the lung may improve the safety and accuracy of VATS segmentectomy comparing with two-dimensional CT images. Purpose of this study was to evaluate the effect of $3 \mathrm{D}$ simulation on the outcome of VATS segmentectomy for pulmonary GGO lesions.

\section{Methods}

\section{Patient preparation and examination}

We retrospectively analyzed 68 cases of solitary pulmonary GGO lesions, whom were diagnosed as peripheral and clinical T1aN0M0 stage IA lung cancer, from May 1, 2016 to February 28, 2017 in our institute. Size of all GGO lesions on CT are less than $2 \mathrm{~cm}$ in diameter and the solid portion of the lesions should be less than $50 \%$ (8). The study was approved by the Ethics Committee of our institute (approval number B2017-115). Written informed consent was obtained from all patients before operation. Patient preoperative characteristics and clinical outcomes were reviewed from a prospective database of our department.

All patients were routinely evaluated by pulmonary function test with blood gas analysis, high resolution CT of the chest with contrast and ultrasound or enhanced CT of abdomen preoperatively. Flexible bronchoscopy, brain magnetic resonance imaging (MRI) or bone scintigraphy were also performed if necessary. Positron emission tomography, mediastinoscopy and endobronchial ultrasound-guided transbronchial needle aspiration (EBUSTBNA) were not routinely performed on the basis of no swelling of mediastinal or hilar lymph nodes were revealed according to CT results. Video-assisted thoracoscopic anatomic segmentectomy were performed on those patients with diagnosis as stage cIA lung cancer according to TNM Classification of Malignant Tumors, seventh edition (9).

Our selection criteria for VATS segmentectomy in the present study were as follows: (I) patients with single peripheral GGO lesion considered suspicious for malignancy; (II) the lesion $<2 \mathrm{~cm}$ in diameter and with at least one of the followings: (i) pure adenocarcinoma in situ (AIS) histology; (ii) nodule has $\geq 50 \%$ ground-glass appearance on high resolution CT; and (iii) radiologic surveillance conforms a long doubling time ( $\geq 400$ days) (2). The patients with insufficient cardiopulmonary function or other contraindication for segmentectomy were excluded.

The pathologic results of the GGO lesions were classified according to 2011 IASLC/ATS/ERS classification (10). The postoperative complications in this study was defined as grade 2 or above according to the Clavien-Dindo classification system (11). The postoperative hospital stay and the 90-day mortality were also recorded.

\section{$3 D$ simulation}

We divided the patients into $3 \mathrm{D}$ group (including 36 patients) and non-3D group (including 32 patients). All patients underwent preoperative contrast-enhanced CT with a slice thickness of $1.0 \mathrm{~mm}$. In non-3D group, we made the diagnosis and the surgical plan according to 

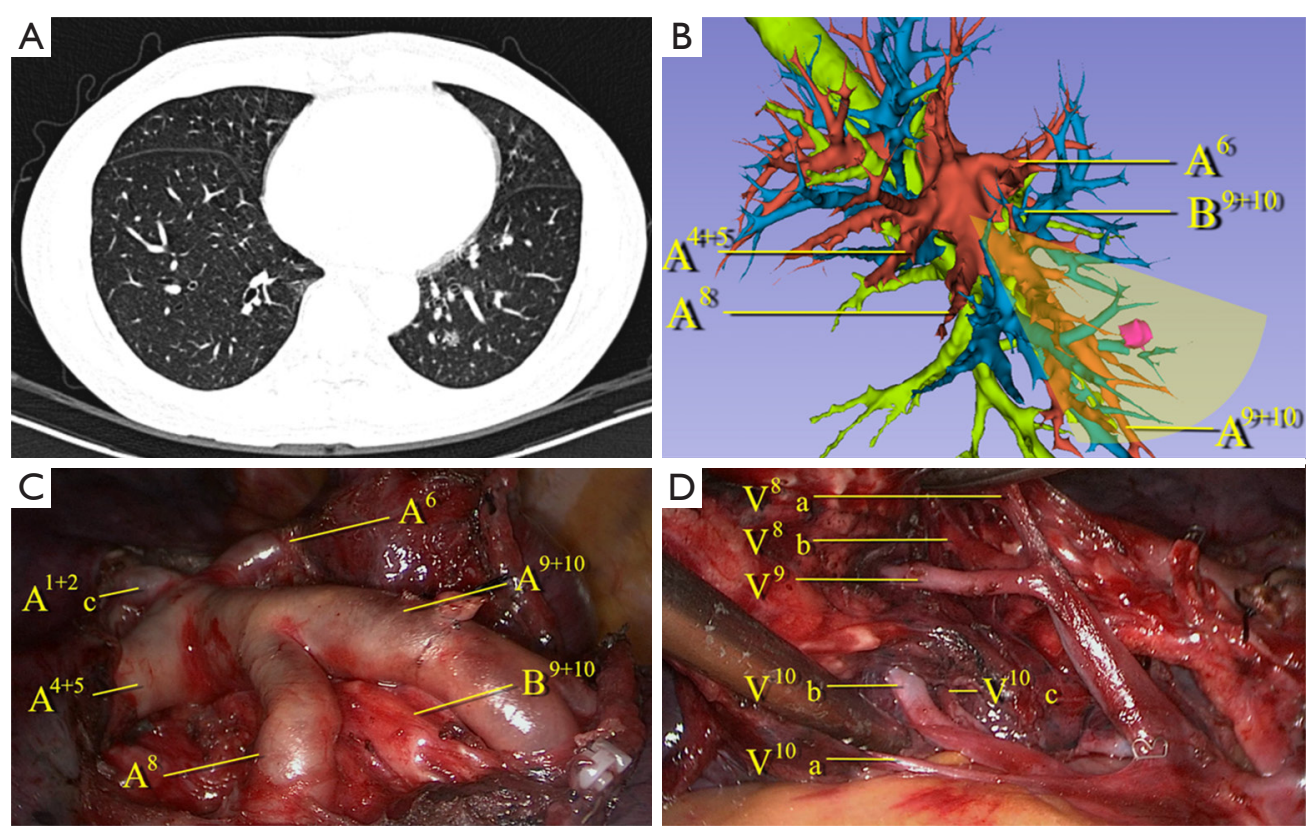

Figure 1 The CT scan and 3D simulation of a GGO lesion in the left lower lobe of a 60-year-old woman. (A) CT scan showed a small GGO lesion in the left $\mathrm{S}^{10}$; the yellow arrowhead indicated the location of the GGO; (B) 3D simulation of the left lung. The fan-shaped area indicated planned resection region $\left(\mathrm{S}^{9}+\mathrm{S}^{10}\right) ;(\mathrm{C})$ the branches of the pulmonary artery were exposed in the VATS segmentectomy for the same patient; (D) the branches of the pulmonary vein were exposed in the VATS segmentectomy procedure. GGO, ground glass opacity; VATS, video-assisted thoracoscopic surgery.

the serial CT images. In 3D group, digital imaging and communications in medicine (DICOM) data of each case was recorded. The method used to reconstruct $3 \mathrm{D}$ images was similar with the previous reports $(6,12,13)$. Volume data from both the arterial and venous phases were obtained at a slice thickness of $1.0 \mathrm{~mm}$. Volume rendering method was used during the reconstruction process. The data was then transferred to a personal computer and converted to a $3 \mathrm{D}$ format by the free software 3D Slicer (version 4, an open source software available on multiple operating systems). The validity of the reconstructions was confirmed and the images were preoperatively evaluated by the thoracic surgeons.

In general, we tended to use $3 \mathrm{D}$ reconstruction in the cases with GGO lesions which may be difficult to locate and the cases with suspected anatomic variations.

The target segment and the surgical plan were firstly decided by CT. All segmental vessels and bronchi to the target segment were then scrutinized on the $3 \mathrm{D}$ images (Figures 1,2). Safe resection margins were identified for each case depending on the distance of vessels and bronchi to the GGO lesion. The safe resection margin was defined as a sphere, extending $2 \mathrm{~cm}$ outside the primary tumor. The center of the margin was manually located to the center of the tumor in the 3D image (6). In 3D group, we identified the relationship between the margin and the intersegmental veins and used this to plan the minimal extent of segmentectomy for every case. The original surgical plans would be changed and enlarged to adjacent segment if the intersegmental veins ran through the safety margin (6).

\section{VATS segmentectomy procedure}

The VATS segmentectomy was performed in all patients via a three-port approach. After intubation, the patients were placed in the lateral decubitus position with onelung ventilation. An observation port was made in the 8th intercostal space on the mid axillary line. Another incision of about $2 \mathrm{~cm}$ was made in the fourth or fifth intercostal space on the anterior axillary line and an incision of about $1.5 \mathrm{~cm}$ was made in the fifth intercostal space on the posterior axillary line.

During the process, the target segmental bronchial and vascular structures were individually isolated and divided. After dissecting and division the bronchus, the distal stump of the target segmental bronchus was lifted 

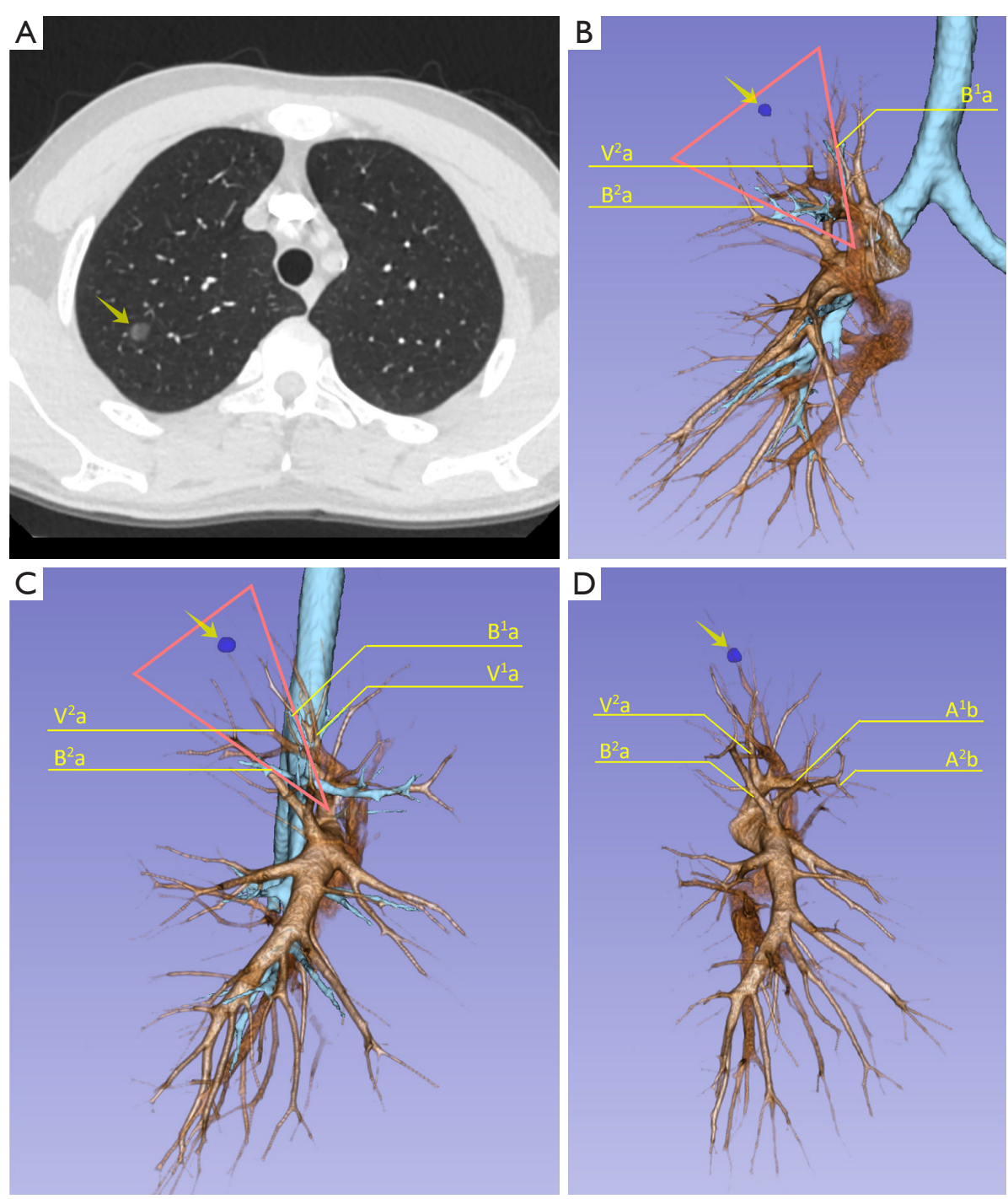

Figure 2 The CT scan and 3D simulation of a GGO lesion in the right upper lobe. (A) CT scan showed a GGO lesion (yellow arrow) in the right upper lobe; (B) anterior view of the 3D simulation image showing the lesion between the $\mathrm{S}^{1}$ a and $\mathrm{S}^{2}$ a. Apical vein without central vein: $\mathrm{V}^{1}$ and $\mathrm{V}^{2}$ form common branch; (C) lateral view of the 3D simulation image showing the lesion between the $\mathrm{S}^{1}$ a and $\mathrm{S}^{2}$ a; (D) branches of segmental arteries: omitting the tracheal images. GGO, ground glass opacity. The red triangle showed the adequate resection margins of this case.

and denuded in the peripheral direction. The whole lung was re-inflated with pure oxygen and then deflated. About 15 minutes later, the inflation-deflation lines became clear to indicate the anatomical intersegmental plane. We may use the staplers, electrocauterization or combination of the facilities to complete the parenchymal transection along the intersegmental plane. The resection line can also be placed on the adjacent segment to the target segment in consideration of sufficient resection margin. If the frozensection diagnosis of malignancy was made to the lesions, sampling or dissection of segmental, lobar, hilar and mediastinal lymph nodes were performed. Fibrin sealant may be used to prevent air leakage if necessary.

\section{Statistical analysis}

Statistical analyses were performed by SPSS 19.0 software (SPSS, Inc., Chicago, IL, USA). Variables were analyzed as means with standard deviation or medians according to their nature.

Mean values were compared using Student's $t$-test and frequency distributions were compared using the Chi- 
Table 1 Preoperative clinicopathological factors of the patients

\begin{tabular}{|c|c|c|c|}
\hline Characteristic & Non-3D $(n=32)$ & $3 D(n=36)$ & $P$ value \\
\hline Age, year & $51.6 \pm 10.5$ & $53.0 \pm 11.1$ & 0.06 \\
\hline \multicolumn{4}{|l|}{ Sex } \\
\hline Men & $12(38 \%)$ & $9(25 \%)$ & 0.30 \\
\hline Women & $20(63 \%)$ & 27 (75\%) & \\
\hline BMI & $21.2 \pm 2.4$ & $20.8 \pm 1.9$ & 0.35 \\
\hline \multicolumn{4}{|l|}{ Image characteristics } \\
\hline Size, mm & $12.5 \pm 3.5$ & $12.0 \pm 2.7$ & 0.44 \\
\hline Pure GGO & $11(34 \%)$ & $13(36 \%)$ & 1.00 \\
\hline GGO with $<50 \%$ solid part & $21(66 \%)$ & $23(64 \%)$ & \\
\hline \multicolumn{4}{|c|}{ Pulmonary function, mean \%pred } \\
\hline FEV1 & $89.9 \pm 12.5$ & $85.0 \pm 18.9$ & 0.20 \\
\hline MVV & $84.6 \pm 9.3$ & $88.6 \pm 8.7$ & 0.06 \\
\hline DLCO & $84.4 \pm 17.9$ & $82.0 \pm 16.6$ & 0.54 \\
\hline Active smokers & $9(28 \%)$ & $12(33 \%)$ & 0.79 \\
\hline \multicolumn{4}{|l|}{ Comorbidity } \\
\hline Hypertension & $3(9 \%)$ & $5(14 \%)$ & 0.80 \\
\hline Diabetes mellitus & $2(6 \%)$ & $4(11 \%)$ & \\
\hline Cancer history & $1(3 \%)$ & 0 & \\
\hline Heart diseases & $3(9 \%)$ & $2(6 \%)$ & \\
\hline $\begin{array}{l}\text { Non-thoracic surgery } \\
\text { history }\end{array}$ & 2 (6\%) & $3(8 \%)$ & \\
\hline Total & $11(34 \%)$ & 14 (39\%) & \\
\hline
\end{tabular}

GGO, ground glass opacity; SD, standard deviation; BMI, body mass index; \%pred, predicted value ratio; DLCO, diffusion capacity of the lung for carbon monoxide; FEV1, forced expiratory volume in 1 second; MVV, maximum voluntary ventilation.

square test or Fischer's exact test. A P value of $<0.05$ was regarded as significant.

\section{Results}

The preoperative patient characteristics are listed in Table 1 . Sixty-eight patients with single peripheral GGO in lung were analyzed. Among them, 21 (31\%) male and 47 (69\%) female were included, aging from 34 to 72 years (median 57).

Forty-five lesions were detected by regular medical examination, 15 lesions were found with respiratory symptoms and 8 lesions were diagnosed occasionally with other diseases. Twenty-four (35\%) lesions were pure GGO.
Table 2 Types of VATS segmentectomy procedure in non-3D group and $3 \mathrm{D}$ group

\begin{tabular}{|c|c|c|}
\hline $\begin{array}{l}\text { Types of segmentectomy } \\
\text { procedure }\end{array}$ & Non-3D & $3 \mathrm{D}$ \\
\hline Left upper lobe & $12(38 \%)$ & 13 (36\%) \\
\hline $\mathrm{S}^{1+2}$ & 5 & 4 \\
\hline$S^{3}$ & 3 & 3 \\
\hline$S^{4+5}$ & 2 & 2 \\
\hline$S^{1+2+3}$ & 2 & 4 \\
\hline Left lower lobe & $5(16 \%)$ & $5(14 \%)$ \\
\hline$S^{6}$ & 3 & 2 \\
\hline$S^{8}$ & 0 & 1 \\
\hline$S^{9+10}$ & 1 & 2 \\
\hline $\mathrm{S}^{8+9+10}$ & 1 & 0 \\
\hline Right upper lobe & $10(31 \%)$ & $12(33 \%)$ \\
\hline$S^{1}$ & 5 & 4 \\
\hline$S^{2}$ & 3 & 4 \\
\hline$S^{3}$ & 2 & 3 \\
\hline$S^{1} a+S^{2} a$ & 0 & 1 \\
\hline Right lower lobe & $5(16 \%)$ & $6(17 \%)$ \\
\hline$S^{6}$ & 3 & 2 \\
\hline$S^{7+8}$ & 1 & 2 \\
\hline$S^{9+10}$ & 1 & 2 \\
\hline
\end{tabular}

Solid ratio of $44(65 \%)$ lesions is less than $50 \%$.

All patients were examined by the followed-up chest CT scan in the course from 10 to 36 months (median 17.5 months). The suspicious diagnosis of lung cancer was made for enlargement of the size or solid part of the lesion in the follow-up period.

Type of the segmentectomy procedures for the two groups were showed in Table 2. 3D reconstruction was successfully built in all cases of 3D group. The original surgical plans of 7 cases (19\%) in 3D group were changed according to the simulation results with the consideration of adequate resection margin distance. Three cases with $<1 \mathrm{~cm}$ GGO lesions extended the resection to the adjacent segment. Two cases of $\mathrm{LS}^{3}$ and one $\mathrm{LS}^{1+2}$ segmentectomy adjusted into $\mathrm{LS}^{1+2+3}$ segmentectomy. One $\mathrm{RS}^{1}$ adjusted into $\mathrm{RS}^{1} \mathrm{a}+\mathrm{S}^{2}$ a segmentectomy.

Adequate resection margin distance was found for all cases in $3 \mathrm{D}$ group. Inadequate resection margin distance 
Table 3 Intraoperative and postoperative characters of non-3D group and 3D group

\begin{tabular}{|c|c|c|c|}
\hline Characters & None-3D & $3 D$ & $P$ value \\
\hline \multicolumn{4}{|l|}{ Adhesion } \\
\hline Mild & $1(3 \%)$ & $3(8 \%)$ & 0.71 \\
\hline Moderate & $2(6 \%)$ & $2(6 \%)$ & \\
\hline \multicolumn{4}{|l|}{ Segmental plane } \\
\hline Stapler & $27(84 \%)$ & $30(83 \%)$ & 1.00 \\
\hline Electrocautery + stapler & $5(16 \%)$ & $6(17 \%)$ & \\
\hline \multicolumn{4}{|l|}{ Postoperative complications } \\
\hline Atrial fibrillation & 0 & $1(3 \%)$ & \\
\hline Air leakage (>7 days) & $2(6 \%)$ & $3(8 \%)$ & \\
\hline Pneumonia & $1(3 \%)$ & 0 & \\
\hline Atelectasis & $1(3 \%)$ & $1(3 \%)$ & \\
\hline Others & $1(3 \%)$ & $1(3 \%)$ & \\
\hline Total & $5(16 \%)$ & $6(17 \%)$ & 1.00 \\
\hline \multicolumn{4}{|l|}{ Histology } \\
\hline Invasive adenocarcinoma & $9(28 \%)$ & $8(22 \%)$ & \\
\hline Microinvasive adenocarcinoma & $18(56 \%)$ & $23(64 \%)$ & \\
\hline Adenocarcinoma in situ & $5(16 \%)$ & $5(14 \%)$ & 0.81 \\
\hline Operation time [range] & $\begin{array}{c}139 \\
{[108-159]}\end{array}$ & $\begin{array}{c}111 \\
{[82-133]}\end{array}$ & 0.03 \\
\hline Intraoperative bleeding, $\mathrm{mL}$ & $129.1 \pm 79.2$ & $106.4 \pm 59.2$ & 0.06 \\
\hline Chest tube duration, days & $4.1 \pm 2.2$ & $4.1 \pm 1.8$ & 0.93 \\
\hline Postoperative hospital stay, days & $5.1 \pm 2.2$ & $5.3 \pm 2.1$ & 0.81 \\
\hline
\end{tabular}

was found for the 4 cases (13\%) in non-3D group. Wedge resections of more lung tissues than the original plan were then performed on these patients $(\mathrm{P}=0.04)$.

The intra-operative and postoperative characters of the patients were showed in Table 3. The complications of the procedures and the dealing of the intersegmental plane were similar in the two groups. There were 4 cases of mild to moderate adhesion in either group. The blood loss, postoperative hospital stay and the incidence of the postoperative complications were similar in both groups. No lymph node metastasis was detected in all the patients. There was no postoperative 30-day mortality in either group. Median operation time for $3 \mathrm{D}$ group (111 minute) was shorter than non-3D group (139 minute) $(\mathrm{P}=0.03)$.

\section{Discussion}

D'Amico and his colleagues reported the technical considerations and showed that thoracoscopic segmentectomy was feasible, which also achieved equivalent oncological results compared with open segmentectomy $(14,15)$. The feasibility of segmentectomy for lung cancer with GGO in patients with adequate lung function for lobectomy has been discussed by Iwata et al. in a retrospective study (16). Most of our patients in the study were in sound condition with adequate lung function before surgery, since most of the small GGO lesions were detected in routine medical examination. All of our patients have received intensively radical segmentectomy according to the NCCN guidelines for NSCLC (2). Incidence of complications for the two groups in the study was similar and rare. There was no thoracotomy or postoperative 90-day mortality in either group. All the patients received regular follow-up chest CT scan after the first detection of the lesions. The diagnosis of lung cancer was made when the lesions enlarged or the solid component developed (17). This may explain why all the cases were malignant in the study.

We adapted an open source software 3D slicer for the reconstruction and it took 10 to 15 minutes for each case. The overview and the application in pulmonary medicine of the software was reported previously by Cheng et al. (18). We found it was also reliable in pulmonary surgical plan. $3 \mathrm{D}$ reconstructions accomplished in all cases of $3 \mathrm{D}$ group in this study. In the present study, most of the GGO lesions were not directly visible in the procedure and were difficult to locate by palpation. We did not use preoperative marking methods described by the previous literatures (19-21) for these GGO lesions. Although these methods are simple for wedge resection, they may not be convenient to directly describe the target segments that contain the lesions. Kato et al. reported thoracoscopic segmentectomy using 3D reconstruction for non-palpable, visually undetectable tumors (22). We also found that $3 \mathrm{D}$ reconstruction is convenient for pulmonary nodule and target segment locating. All of the GGO lesions were resected in the target segments in our study. Comparing with the twodimensional CT images, we found 3D simulation was very helpful for precise resection GGO lesions located in particularly deep parenchyma or on border of the adjacent segments (Figure 1). On 3D simulation images, the lesion and the target segment were determined according to the relationship between the GGO lesion and the whole view of segmental vessels and bronchus. Also the relationship 
between the lesions and the segmental border can be identified directly to preclude the misjudgment of the target segment.

In our study, we inspected the surgical margins in each case after the specimen was resected. Intraoperative frozen section diagnosis was performed in every case. Additional wedge resection would be performed using staplers in cases with margin distance less than $2 \mathrm{~cm}$ or two times of the diameter of the GGO lesions. There were 4 cases of insufficient resection margins in the present study and all of them were in non-3D group. This may be partly due to pulmonary collapse during operation, reducing the enough margin distance. However, it was also difficult in some cases to identify all the vessels and bronchi involved in threedimensional space that should be removed according to two dimensional CT images. It may rely on the personal experience of the surgeons to avoid this kind of risk. In 3D group, the reconstructed images can be rotated freely and visualized from any angle, the $3 \mathrm{D}$ relationships and the distance of the vessels and bronchi with the GGO lesions can be measured directly. When we facilitated 3D simulation in these cases, we found it was convenient for us to tell which pulmonary segment or segments that involved these structures should be removed. According to the $3 \mathrm{D}$ images, surgical plans of 7 patients were adjusted. Three cases with $<1 \mathrm{~cm}$ GGO lesions extended the resection to the adjacent segment. Two cases of $\mathrm{LS}^{3}$ segmentectomy and one $\mathrm{LS}^{1+2}$ changed into $\mathrm{LS}^{1+2+3}$ segmentectomy. One $\mathrm{RS}^{1}$ turned into $\mathrm{RS}^{1} \mathrm{a}+\mathrm{S}^{2}$ a segmentectomy. That precluded the insufficient resection distance in the procedures and we did not have to preform any additional wedge resection or lobectomy in 3D group. This indicated that $3 \mathrm{D}$ simulation can be useful in reducing the risk of insufficient surgical margin.

Segmentectomy is technically more complicated than lobectomy due to the anatomical complexity and variations of peripheral vessels and bronchi. The previous reports of $3 \mathrm{D}$ reconstruction of different pulmonary lobe variation condition indicated that these variations were common and had many different patterns $(23,24)$. In order to make sure the accuracy and security of the segmentectomy procedure, we need to confirm the correctness before division any branch of blood vessels and bronchi. Sometimes it may also take time for extended biopsy of the lung tissue and resulting in more operation time. In our study, the operation time for 3D group was significantly shorter than non-3D group. The surgeons can understand precisely the topological structure of vessels and bronchi before the surgery by studying $3 \mathrm{D}$ reconstruction images thoroughly.
The anatomic variations were directly found prior to surgery in $3 \mathrm{D}$ group (Figure 1). In the normal pattern of the distribution of the vessels and bronchi, unnecessary dissection of lung tissue was avoided. This may explain the reason of less operation time in $3 \mathrm{D}$ group.

In the present study there were some limitations. It was retrospectively designed. The number of the cases was also small. Our results showed the merits of $3 \mathrm{D}$ reconstruction in VATS segmentectomy and this was consistent with the previous literatures $(7,22,25)$. Certainly, prospective studies with larger number of the cases are warranted to verify the advantages of $3 \mathrm{D}$ reconstruction in VATS segmentectomy for treatment of GGO.

In conclusion, the guidance of $3 \mathrm{D}$ simulation images may enable accurate locate of the GGO lesions and illustrate the variation pattern of the segmental vessels and bronchi. $3 \mathrm{D}$ preoperative simulation can be more helpful in precise surgical plan than CT scan and significantly shorten the operation time in VATS segmentectomy for GGO in lung.

\section{Acknowledgements}

None.

\section{Footnote}

Conflicts of Interest: This study was presented at the 31st EACTS Annual Meeting, 7-11 October 2017, in Vienna, Austria.

Ethical Statement: The study was approved by the Ethics Committee of our institute (approval number B2017-115). Written informed consent were obtained from all patients before operation.

\section{References}

1. Zhang Y, Sun Y, Wang R, et al. Meta-analysis of lobectomy, segmentectomy, and wedge resection for stage I nonsmall cell lung cancer. J Surg Oncol 2015;111:334-40.

2. Ettinger DS, Akerley W, Bepler G, et al. Non-small cell lung cancer. J Natl Compr Canc Netw 2010;8:740-801.

3. Sagawa M, Oizumi H, Suzuki H, et al. A prospective 5-year follow-up study after limited resection for lung cancer with ground-glass opacity. Eur J Cardiothorac Surg 2018;53:849-56.

4. Zhang L, Li M, Yin R, et al. Comparison of the oncologic outcomes of anatomic segmentectomy and lobectomy for 
early stage non-small cell lung cancer. Ann Thorac Surg 2015;99:728-37.

5. Bao F, Ye P, Yang Y, et al. Segmentectomy or lobectomy for early stage lung cancer: a meta-analysis. Eur J Cardiothorac Surg 2014;46:1-7.

6. Iwano S, Yokoi K, Taniguchi T, et al. Planning of segmentectomy using three-dimensional computed tomography angiography with a virtual safety margin: technique and initial experience. Lung Cancer 2013;81:410-5.

7. Chan EG, Landreneau JR, Schuchert MJ, et al. Preoperative (3-dimensional) computed tomography lung reconstruction before anatomic segmentectomy or lobectomy for stage I non-small cell lung cancer. J Thorac Cardiovasc Surg 2015;150:523-8.

8. Schuchert MJ, Abbas G, Pennathur A, et al. Sublobar resection for early-stage lung cancer. Semin Thorac Cardiovasc Surg 2010;22:22-31.

9. Goldstraw P, Crowley J, Chansky K, et al. The IASLC Lung Cancer Staging Project: proposals for the revision of the TNM stage groupings in the forthcoming (seventh) edition of the TNM Classification of malignant tumours. J Thorac Oncol 2007;2:706-14.

10. Travis WD, Brambilla E, Noguchi M, et al. International association for the study of lung cancer/american thoracic society/european respiratory society international multidisciplinary classification of lung adenocarcinoma. J Thorac Oncol 2011;6:244-85.

11. Dindo D, Demartines N, Clavien PA. Classification of surgical complications: a new proposal with evaluation in a cohort of 6336 patients and results of a survey. Ann Surg 2004;240:205-13.

12. Gossot D, Lutz J, Grigoroiu M, et al. Thoracoscopic anatomic segmentectomies for lung cancer: technicalaspects. J Vis Surg 2016;2:171.

13. Yao F, Wang J, Yao J, et al. Three-dimensional image reconstruction with free open-source OsiriX software in video-assisted thoracoscopic lobectomy and segmentectomy. Int J Surg 2017;39:16-22.

14. D'Amico TA. Thoracoscopic segmentectomy: technical considerations and outcomes. Ann Thorac Surg 2008;85:S716-8.

15. Yang CF, D'Amico TA. Thoracoscopic segmentectomy for lung cancer. Ann Thorac Surg 2012;94:668-81.

16. Iwata H, Shirahashi K, Mizuno Y, et al. Feasibility of segmental resection in non-small-cell lung cancer with ground-glass opacity. Eur J Cardiothorac Surg 2014;46:375-9; discussion 379.
17. Gould MK, Donington J, Lynch WR, et al. Evaluation of individuals with pulmonary nodules: when is it lung cancer? Diagnosis and management of lung cancer, 3rd ed: American College of Chest Physicians evidence-based clinical practice guidelines. Chest 2013;143:e93S-e120S.

18. Cheng GZ, San Jose Estepar R, Folch E, et al. Threedimensional Printing and 3D Slicer: Powerful Tools in Understanding and Treating Structural Lung Disease. Chest 2016;149:1136-42.

19. Kerrigan DC, Spence PA, Crittenden MD, et al. Methylene blue guidance for simplified resection of a lung lesion. Ann Thorac Surg 1992;53:163-4.

20. Ikeda K, Nomori H, Mori T, et al. Impalpable pulmonary nodules with ground-glass opacity: Success for making pathologic sections with preoperative marking by lipiodol. Chest 2007;131:502-6.

21. Bolton WD, Howe H 3rd, Stephenson JE. The utility of electromagnetic navigational bronchoscopy as a localization tool for robotic resection of small pulmonary nodules. Ann Thorac Surg 2014;98:471-5; discussion 475-6.

22. Kato H, Oizumi H, Suzuki J, et al. Thoracoscopic anatomical lung segmentectomy using 3D computed tomography simulation without tumour markings for nonpalpable and non-visualized small lung nodules. Interact Cardiovasc Thorac Surg 2017;25:434-41.

23. Nagashima T, Shimizu K, Ohtaki Y, et al. An analysis of variations in the bronchovascular pattern of the right upper lobe using three-dimensional CT angiography and bronchography. Gen Thorac Cardiovasc Surg 2015;63:354-60.

24. Shimizu K, Nagashima T, Ohtaki Y, et al. Analysis of the variation pattern in right upper pulmonary veins and establishment of simplified vein models for anatomical segmentectomy. Gen Thorac Cardiovasc Surg 2016;64:604-11.

25. Wu WB, Xu XF, Wen W, et al. Three-dimensional computed tomography bronchography and angiography in the preoperative evaluation of thoracoscopic segmentectomy and subsegmentectomy. J Thorac Dis 2016;8:S710-5.

Cite this article as: Xue L, Fan H, Shi W, Ge D, Zhang Y, Wang Q, Yuan Y. Preoperative 3-dimensional computed tomography lung simulation before video-assisted thoracoscopic anatomic segmentectomy for ground glass opacity in lung. J Thorac Dis 2018;10(12):6598-6605. doi: 10.21037/ jtd.2018.10.126 